\title{
MiR-608 regulating the expression of ribonucleotide reductase M1 and cytidine deaminase is repressed through induced gemcitabine chemoresistance in pancreatic cancer cells
}

\author{
Azam Rajabpour ${ }^{1,2,3} \cdot$ Ali Afgar $^{2} \cdot$ Habibollah Mahmoodzadeh ${ }^{4} \cdot$ Jalal-e-Din Radfar $^{5}$. \\ Farzad Rajaei $^{1,3} \cdot$ Ladan Teimoori-Toolabi $^{2}$
}

Received: 31 January 2017 / Accepted: 7 August 2017 / Published online: 8 September 2017

(c) Springer-Verlag GmbH Germany 2017

\begin{abstract}
Purpose Gemcitabine resistance is the main problem in pancreatic adenocarcinoma patients. Hence, we aimed to identify the correlation between expression of RRM1 and CDA as the resistance genes and their predicted targeting miR-608 in the resistant pancreatic cancer cell lines to gemcitabine.

Methods Dual luciferase assay was performed to determine whether both RRM1 and CDA are targeted by miR-608 in 293T and pancreatic cancer cell lines. AsPC-1 and MIA $\mathrm{PaCa}-2$ cell lines became gradually resistant to gemcitabine by exposing to the increasing doses of gemcitabine. After RNA and miRNAs extraction and cDNA conversion, the expressions of RRM1, CDA and miR-608 in all cell lines were studied by quantitative PCR. Pre-miR- 608 transfection to the cell lines was done by calcium phosphate method. MTT assay was performed for analyzing the chemo sensitivity of different cell lines to gemcitabine.
\end{abstract}

Farzad Rajaei

farzadraj@yahoo.co.uk

$\triangle$ Ladan Teimoori-Toolabi

lteimoori@pasteur.ac.ir

1 Department of Molecular Medicine, School of Medicine, Qazvin University of Medical Sciences, Qazvin, Iran

2 Molecular Medicine Department, Pasteur Institute of Iran, Tehran, Iran

3 Cellular and Molecular Research Center, Qazvin University of Medical Sciences, Qazvin, Iran

4 Cancer Institute of Iran, Imam Khomeini Medical Complex, Tehran University of Medical Sciences, Tehran, Iran

5 National Cell Bank of Iran, Pasteur Institute of Iran, Tehran, Iran
Results Luciferase assays showed that miR-608 targeted RRM1 and CDA genes in 293T, AsPC-1 and MIA PaCa-2 cell lines. Compared to parental cell line, resistant MIA PaCa-2 and AsPC-1 cells demonstrated increased expression of RRM1 and CDA. On the other hand the expression of miR-608 in resistant MIA PaCa-2 and AsPC- 1 cells was lower than parental cells. Furthermore, transfection of MIA PaCa- 2 and AsPC- 1 cells by miR-608 lead to decreased expression of RRM1 and CDA and lowered viability of the cells in comparison with scrambled microRNA transfected cells.

Conclusion During resistance induction in pancreatic cancer cells, miR-608 which is targeting RRM1 and CDA is downregulated which leads to upregulation of these genes.

Keywords Pancreatic neoplasms - Gemcitabine resistance $\cdot$ RRM1 protein, human · CDA · MIRNA-608, microRNA, human

$\begin{array}{ll}\text { Abbreviations } \\ \text { PDAC } & \text { Pancreatic adenocarcinoma } \\ \text { GEM } & \text { Gemcitabine } \\ \text { CDA } & \text { Cytidine deaminase } \\ \text { RRM1 } & \text { Ribonucleotide reductase M1 } \\ \text { dNTP } & \text { Deoxynucleoside triphosphate } \\ \text { miR } & \text { miRNA, microRNA } \\ \text { dFdU } & \text { 2'2'-Difluorodeoxyuridine } \\ \text { IC } & \text { Inhibitory concentration } \\ \text { MTT } & \text { 3-(4,5-Di methylthiazole-2-yl)-2, 5-biphenyl } \\ & \text { tetrazolium bromide }\end{array}$

Abbreviations

PDAC Pancreatic adenocarcinoma

GEM Gemcitabine

CDA Cytidine deaminase

RRM1 Ribonucleotide reductase M1

dNTP Deoxynucleoside triphosphate

miR miRNA, microRNA

dFdU 2'2'-Difluorodeoxyuridine

IC Inhibitory concentration

tetrazolium bromide 